\title{
Staphylococcal pneumonia, an actual challenge in pediatric practice - a case report
}

\author{
Nicoleta-Ana Tomsa', Lorena Elena Melit ${ }^{1,2}$, Gabriela Bucur', Iunius Simu ${ }^{2,3}$, \\ Bianca Aron', Cristina Oana Marginean ${ }^{1,2}$ \\ ${ }^{1}$ Pediatrics Clinic, Emergency Clinical County Hospital, Tg. Mures, Romania \\ 2"G.E. Palade" University of Medicine, Pharmacy, Sciences and Technology, Tg. Mures, Romania \\ ${ }^{3}$ Radiology and Medical Imaging Clinic, Emergency Clinical County Hospital, Tg. Mures, Romania
}

\begin{abstract}
Introduction. Approximately 12 million children die every year in developing countries and one third of these deaths are caused by pneumonias. The assessment of radiological changes in dynamics owns a major role in establishing the diagnosis of staphylococcal pneumonia.

The aim of this paper was to underline the diagnostic and treatment difficulties in a child with staphylococcal pneumonia.

Case report. We present the case of a 2 years and 6-month-old male patient admitted in our clinic for hyperpyrexia, productive cough, rhinorrhea and anorexia. The clinical exam at admission pointed out influenced general status, pale ailing face, pallor, fever, productive cough, rhinorrhea, diminished breath sound on the right side, oxygen saturation $97 \%$ and anorexia, while de blood tests revealed severe leukocytosis with neutrophilia, anemia, and severely increased inflammatory biomarkers. The chest X-ray showed an opacity within the right inferior lobe. The blood culture was negative. We initiated symptomatic and antibiotic treatment (Ceftriaxone), but due to the unfavorable evolution, the control chest X-ray showed a consolidation area within the right inferior lobe and secluded basal pleural effusion as well as on the right lateral side. We performed also a chest computed tomography (CT), noticing a pneumonic process within two segments of the inferior right lobe, several grouped pneumatocele below the pleura, associating within both lungs areas of lobular hyperinflation. Thus, we raised the suspicion of staphylococcal pneumonia, and we initiated Vancomycin by vein, with favorable evolution.

Conclusions. Staphylococcal pneumonia is a well-defined and described pathology in the literature, but still remains a contemporary pathology imposing multiple difficulties related to the diagnosis and management in pediatric patients.
\end{abstract}

Keywords: child, pneumonia, S. aureus, imagistic assessment

\section{INTRODUCTION}

Staphylococcus aureus (S. aureus) was described for the first time in 1800 as a cause of sepsis and abscesses. Microbiologically, it is a Gram positive, yellow bacterium, presenting as cocci with tendency to gather in groups similar to clusters; these microorganisms being able to grow in both aerobic and anaerobic culture environments, at a temperature between 18 and 40 Celsius degree. Specialized laboratories used particular culture environments in order to differentiate the $S$. aureus species from other species $(1,2,3)$. $S$. aureus causes in most of the cases skin infections, but it is also involved in the etiology of lower respiratory infections (pneumonia), as well as cardiac ones - endocarditis, or bones - osteomyelitis $(1,4)$. Nasal carriage is defined by the presence of this bacterium within the nasal cavity, existing in approximately $30 \%$ of population. The transmission is possible from one person to another, but also through contaminated objects, and rarely through cough or sneezing. The bacteria and viruses are a part 
of the normal populating the upper and lower airways. A potential mechanism in the development of pneumonia is represented by the replication of the existing microorganisms at this level related to the impairment of host's immune system $(1,2)$. In terms of $S$. aureus, it is essential to delineate two entities, methicillin sensitive $S$. aureus (MSSA), and methicillin resistant S. aureus (MRSA), respectively, in order to differentiate the therapy $(2,3)$.

Staphylococcal pneumonia was described for the first time in 1919 during the flu pandemics, and the data from children with this pathology were collected initially between 1950-1960 in Europe and North America $(5,6)$. It is estimated that approximately 12 million children die annually in the developing countries, and one thirds of these deaths are due to pneumonia, frequently determined by Streptococcus pneumoniae and Hemophilus influenzae, S. aureus being involved in $5-10 \%$ of the cases $(6,7)$. It is a severe pathology, encountered most commonly in infant and small child, with a mortality of $12-15 \%$ $(1,8)$.

The diagnosis of staphylococcal pneumonia begins by the patient's evaluation possibly revealing influenced general status, high fever, productive cough, rhinorrhea, anorexia, abdominal pain, vomiting and accelerated bowel movements $(5,6)$. The laboratory tests show leukocytosis with neutrophilia, anemia and elevated inflammatory biomarkers. The blood culture is essential in identifying the etiology, but they are positive in only $20-40 \%$ of the cases (5). The gold standard for pneumonia remains the imagistic assessment, i.e. chest X-ray, that was lately replaced by thoracic ultrasound $(9,10)$. It is extremely important to mention that radiological exam in suspected staphylococcal pneumonia must be performed in dynamics and it might reveal lobular infiltrate, cavities or empyema. In the cases when chest $\mathrm{X}$-ray is not enough for establishing completely the diagnosis, a thoracic computed tomography (CT) is indicated in order to assess more precisely the lobular infiltrates or the existing lesions within the lung (10).

The management of staphylococcal pneumonia is based on both symptomatic and especially the etiologic treatment, which in mild and medium forms involves monotherapy with Vancomycin or Oxacillin by vein, while in severe ones antibiotics associations such as Vancomycin and Oxacillin or Gentamycin in methicillin resistant cases $(1,5)$. A surgical treatment is mandatory in forms associating massive pleural effusion with external drainage of this fluid. The evolution of prognosis in case of this pathology are unpredictable, being a severe condition, burdened by the patient's comorbidities, a poor clinical course and an increased mortality rate (5).

The aim of this case report is to underline the difficulties related to the diagnosis and management in a child with staphylococcal pneumonia.

\section{CASE REPORT}

\section{Reasons of admission}

We report the case of 2 years and 6-month-old male patient, born preterm at 33-34 gestational weeks, admitted in our clinic for hyperpyrexia, productive cough, rhinorrhea and anorexia. We mention that the onset of the symptoms was 6 days before the admission, being treated ambulatory with antibiotic and symptomatic treatment, but without improvement.

\section{Clinical exam}

The clinical exam at the time of admission revealed influenced general status, pallor, fever $\left(38.8^{\circ} \mathrm{C}\right)$, productive cough, rhinorrhea, diminished breath sounds on the right lung, oxygen saturation $97 \%$, and anorexia. The patient weighed $11 \mathrm{~kg}$.

\section{Diagnostic focus and assessment}

The laboratory tests performed at the time of admission pointed out severe leukocytosis $(21,120 /$ $\mathrm{mm} 3$ ) with neutrophilia (72\%), mildly decreased hemoglobin ( $\mathrm{Hgb} 10,5 \mathrm{~g} / \mathrm{dl}$ ), severely increased inflammatory biomarkers (C-reactive protein - CRP $107 \mathrm{mg} / \mathrm{l}$ and erythrocyte sedimentation rate - ESR $80 \mathrm{~mm} / \mathrm{h}$ ). The chest X-ray pointed out an opacity on the inferior right lobe (Figure 1). The blood culture was negative. We initiated antibiotic (Ceftriaxone by vein) and symptomatic treatment with mildly favorable evolution. After approximately 72 hours from admission, the patient's condition worsened, with fever, the laboratory tests persisting altered (leukocytes 10,250/mm3, neutrophils 24\%, CRP 73,1 mg/l, ESR: $41 \mathrm{~mm} / \mathrm{h}$ ), reason for which we decided to repeat the chest X-ray, which revealed an infiltrate on the lower lobe of the right lung associating also a secluded pleural effusion on the lower and the right side of the thorax. We performed also a thoracic CT following the surgeon's recommendation, noticing pneumonia 


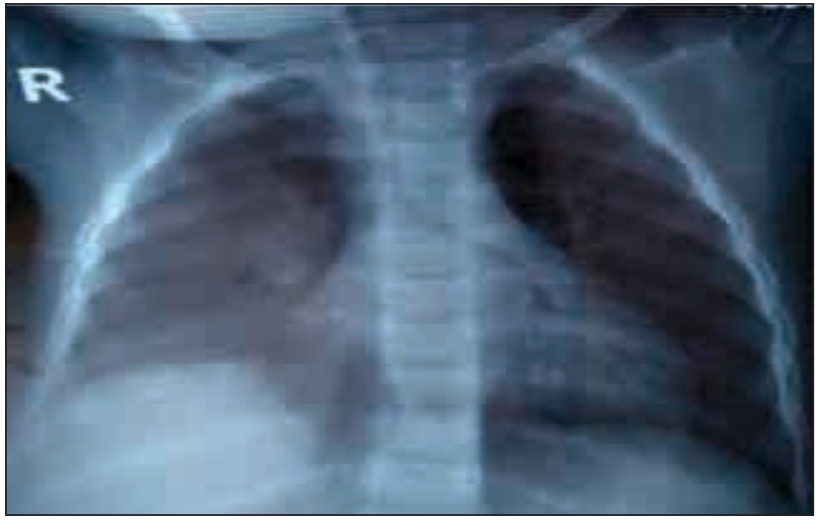

FIGURE 1. Chest $X$-ray at admission - consolidation within the right inferior lobe aspect

aspect within two segments of the inferior right lobe (lateral and posterior) with air bronhogram, several pneumatoceles grouped under the pleura, associating within both lungs areas of lobular hyperinflation (Figure 2). Based on anamnesis, the clinical exam, the undulating evolution and the laboratory tests we considered the most-likely diagnosis to be staphylococcal pneumonia.

\section{Therapeutic focus, assessment and evolution}

We associated Vancomycin by vein, antipyretics (Paracetamol) and rehydration perfusion, with improvement of the general status, fever remission, regaining the appetite, normalization of the laboratory parameters (leukocytes 6,210/mm3, Hgb $11.6 \mathrm{~g} / \mathrm{dl}$, CRP $1.77 \mathrm{mg} / \mathrm{l}$ ), and the chest X-ray performed at 2 weeks after discharge pointed out segmentary/subsegmentary right atelectasis.

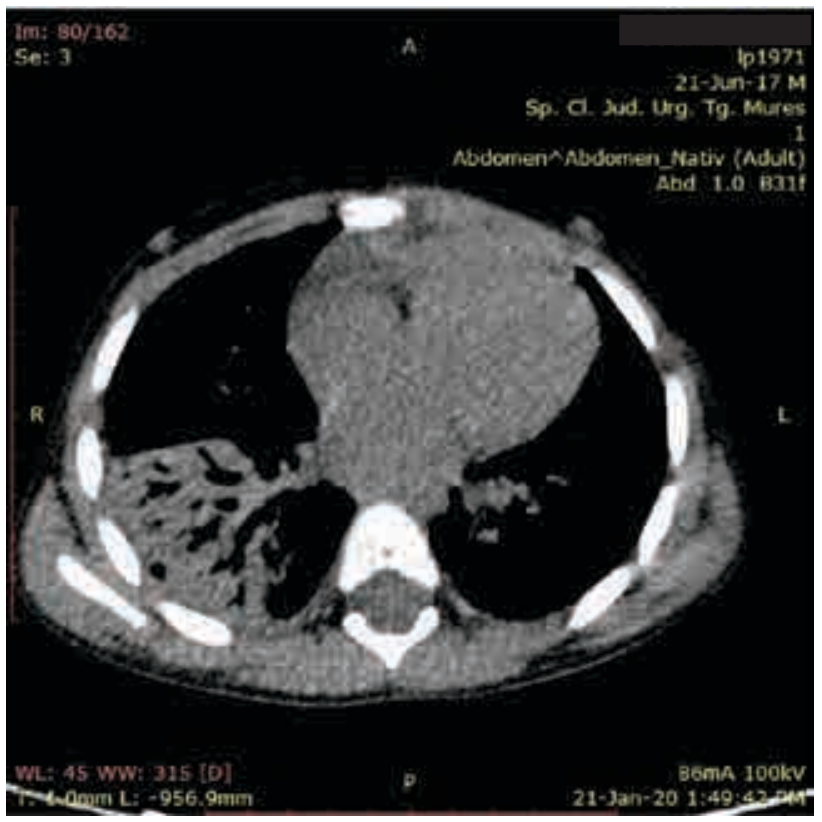

\section{DISCUSSIONS}

Pneumonia and malnutrition are two of the most important causes of mortality during childhood (11). S. aureus is a ubiquitous bacterium in both urban and rural areas. The virulence features of $S$. aureus (i.e. weak immunogenicity, necrotizing, complex toxigenicity, antifungal resistance, the capacity to metastasize and to escape phagocytosis) and neutrophils function temporarily suppressed by a past viral infection are responsible by a severe condition in all age groups with normal cellular and humoral immunity $(12,13)$. We identified no history of recent viral infection in our patient, and the evolution was favorable after the introduction of targeted antibiotic for $S$. aureus. Almost half og the children with staphylococcal bacteriemia might express a disseminated disease. Nevertheless, the recent data from the literature are limited only to isolated case reports (14-16), and the pathology is commonly diagnosed in former healthy children aged between 5-10 years of age $(16,17)$. Male gender is more frequently affected as compared to female one, reflecting the existing gender prevalence in our society (18). Similarly, our patient was a male, but the diagnosis was established much earlier as compared to the mean age reported in the literature, i.e. 2 years and 6 months. The nasal carriage seems to be more important in patient from temperate area (19). Hematogenous dissemination might result in wide metastatic dissemination, especially in children from poor social environments and with malnutrition (20).

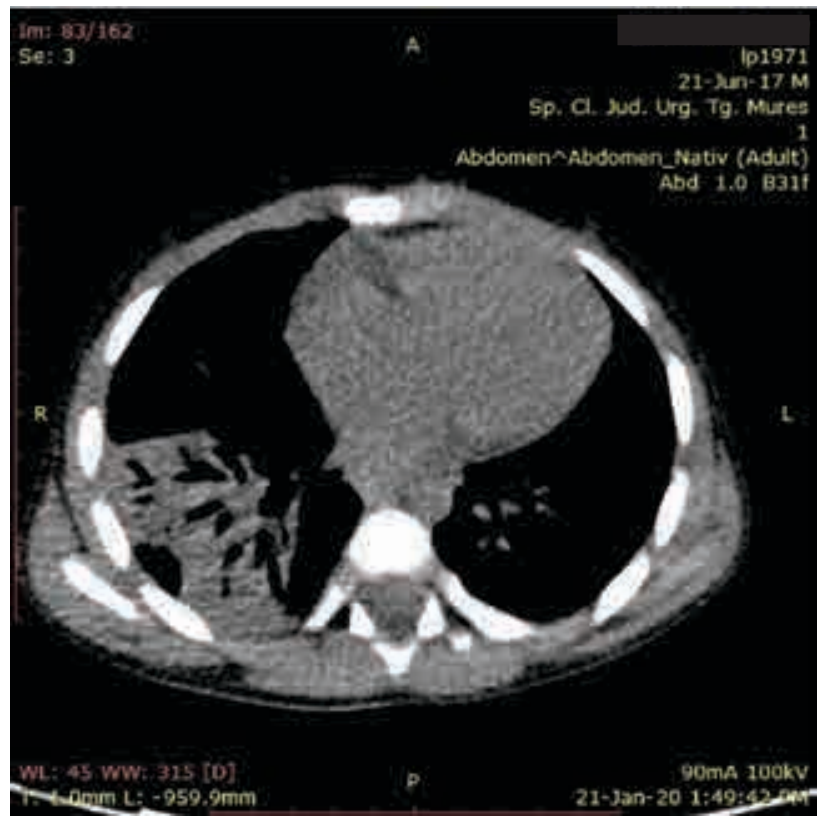

FIGURE 2. Chest CT - pneumonic process affecting two segments in the inferior right lobe, with pneumatocele grouped subpleural, associating within both lungs areas of lobular hyperinflation 
Pleural and lung involvement associated to $S$. aureus represents the most common reason for physician referral $(15,17)$. Staphylococcal pneumonia is manifested by two forms: primary pneumonia due to direct inoculation through airways and secondary metastatic lung infections due to bacterial seeding from other places (13,21-23). Primary pneumonia usually appears during early childhood and in children that associate also malnutrition (13). The patient described by us had most-likely a primary form of staphylococcal pneumonia taking into account the initial symptoms suggesting an upper airways infection and the lack of another infectious situs. Pleural and lung impairment might be manifested by a rapidly progressive condition and might have specific radiological features with a rapid dynamic progressing each day, i.e. multiple, bilateral, nodular, parenchymatous lesions, lung infiltrates, empyema, pneumothorax, pneumatoceles and abscesses (24-26). Similarly, our case presented important radiological changes that were suggestive for the diagnosis, even from the first radiological investigation that showed a right lung infiltrate, as well as the second one which pointed out also pleural effusion, and chest CT which supplementary revealed pneumatoceles.

Admission, preferably in an intensive care unit, is indicated in patients with staphylococcal pneumonia to manage possible emergencies such as septic shock, respiratory insufficiency, tensional pneumothorax or cardiac tamponade $(23,24)$. In our case, the admission in the intensive care unit was not needed since the patient presented no criteria for septic shock or respiratory insufficiency, and diagnosis establishment was early enough to initiate the targeted antibi-

\section{REFERENCES}

1. Bush LM. Staphylococcus aureus infections. Available at: https:// www.msdmanuals.com/home/infections/bacterial-infections-grampositive-bacteria/staphylococcus-aureus-infections.

2. Centers for Disease Control and Prevention (CDC). Outbreaks of community-associated methicillin-resistant Staphylococcus aureus skin infections--Los Angeles County, California, 2002-2003. MMWR Morb Mortal Wkly Rep. 2003 Feb 7;52(5):88.

3. Boucher HW, Corey GR. Epidemiology of methicillin-resistant Staphylococcus aureus. Clin Infect Dis. 2008 Jun 1;46 Suppl 5:S344-9.

4. Lowy FD. Staphylococcus aureus infections. N Engl J Med. 1998;20;339(8):520.

5. Barson WJ. Pneumonia in children: Inpatient treatment. Available at: https://www.uptodate.com/contents/pneumonia-in-children-inpatienttreatment/print,

6. World Health Organization. Technical bases for the WHO recommendation on the management of pneumonia in children at first level health facilities; WHO (ARI) 91.20.World Health Organization, Geneva 1991. otic with major impact on the further evolution of this case. In an analysis performed by Scott and Hall in 2009 that included 509 cases of children with pneumonia who associated also malnutrition, the cause of pneumonia was identified in only $42 \%$ of these cases, blood cultures being considered as an alternative due to the positive result in only $30 \%$ of all patients (11). Moreover, five studies included in this metanalysis used pulmonary aspirate for isolating the pathogen, reporting isolating rates that varied between 25 and $79 \%$ (11). Our case sustains this information since the blood culture did not reveal the pathogen, and the dynamic radiological changes guided our diagnosis.

The identification of the pathogen might be difficult in case of small children with acute community acquired pneumonia. Staphylococcal pneumonia must be suspected when history and clinical exam elements, as well as the evolution and dynamic radiological exam are suggestive in order to initiate as soon as possible a proper treatment.

\section{CONCLUSIONS}

Staphylococcal pneumonia is a childhood pathology whose clinical picture is generally non-specific. Blood cultures are negative in most of the cases, and the need for imagistic assessment at presentation, as well as in dynamics is essential for a proper and early diagnosis. Thus, staphylococcal pneumonia is well defined and well-known pathology in the literature, but still remains a contemporary pathology imposing multiple difficulties related to the diagnosis and management in pediatric cases.

Conflict of interest: none declared Financial support: none declared

7. Chickering HT, James HP Jr. Staphylococcus aureus pneumonia. JAMA. 1919;72(9):617-626.

8. Stefano A, Luis FR, Paola F et al. Global initiative for aureus pneumonia (GLIMP): an international, observational cohort study. Lancet Infect Dis. 2016;16(12):1364-1376.

9. Mandell LA, Wunderink RG, Anzueto A, Bartlett JG, Campbell GD, Dean NC, Dowell SF, File TM Jr, Musher DM, Niederman MS, Torres A, Whitney CG; Infectious Diseases Society of America; American Thoracic Society. Infectious Diseases Society of America/American Thoracic Society consensus guidelines on the management of community-acquired pneumonia in adults. Clin Infect Dis. 2007 Mar 1;44 Suppl 2(Suppl 2):S27-72.

10. Syrjälä H, Broas M, Suramo I, Ojala A, Lähde S. High-resolution computed tomography for the diagnosis of community-acquired pneumonia. Clin Infect Dis. 1998 Aug;27(2):358-63.

11. Chisti MJ, Tebruegge M, La Vincente S, Graham SM, Duke T. Pneumonia in severely malnourished children in developing countries - mortality risk, aetiology and validity of WHO clinical 
signs: a systematic review. Trop Med Int Health. 2009 Oct;14(10):1173-89.

12. O'Driscoll M, Crawford L, Biggar WD. Clinical features and abnormal neutrophil function in disseminated staphylococcal disease. Pediatr Infect Dis. 1985 Mar-Apr;4(2):137-41.

13. Melish ME, Campbell KA. Coagulase-positive staphylococcal infections. In: Feigin RD, Cherry JD, eds. Textbook of infectious diseases. Philadelphia: W.B. Saunders Company. 1998:1039-66.

14. Ladhani S, Konana OS, Mwarumba S et al. Bacteremia due to Staphylococcus aureus. Arch Dis Child. 2004;89:568-571.

15. Suryati BA, Watson M. Staphylococcus aureus bacteraemia in children: a 5-year retrospective review. J Paediatr Child Health. 2002;38:290-4.

16. Kabra SK, Jain YK, Kataria A et al. Disseminated staphylococcal disease. Indian Pediatr. 1996;33:683-5.

17. Hieber JP, Nelson AJ, McCracken GH Jr. Acute disseminated staphylococcal disease in childhood. Am J Dis Child. 1977; 131:181-5.

18. Singhi S, Jain V, Gupta G. Pediatric emergencies in a tertiary care hospital. J Trop Paediatr. 2003;49:207-11.
19. Von EiffC, Becker K, Machka K et al. Nasal carriage as a source of Staphylococcus aureus bacteremia - Study Group. N Engl J Med. 2001;344:11-6.

20. Issac KM, Mbise RL, Hirji KF. Nosocomial bacterial infections among children with severe protein calorie malnutrition. East Afr Med J. 1992:69:433-6.

21. Chartrand SA, McCracken GHJr. Staphylococcal pneumonia in infants and children. Pediatr Infect Dis. 1982;1:19-23.

22. Naraqi S, McDonell G. Hematogenous staphylococcal pneumonia secondary to soft tissue infection. Chest. 1981;79:173-5.

23. Goel A, Bamford L, Hanslo D, et al. Primary staphylococcal pneumonia in young children: a review of 100 cases. J Trop Pediatr. 1999;45:233-6.

24. Popescu V, Dragomir D. Staphylococcal pneumonia. Pediatrie (Bucur). 1991;40:85-106.

25. Hendren WH, Haggerty RJ. Staphylococcal pneumonia in infancy and childhood: analysis of 75 cases. JAMA. 1958;168:6-16.

26. Rebhan AW, Edwards HE. Staphylococcal pneumonia: a review of 329 cases. Can Med Assoc J. 1960;82:513-7. 